\begin{tabular}{lllll} 
Abstract PTH-138 Table 1 & & & \\
\hline Angiogenic factor & Patient mean & Patient range & Control mean & Control range \\
\hline Ang-1 & 13071 & $615-43833$ & 21169 & $2253-56010$ \\
Ang-2 & 4600 & $842-11767$ & 2973 & $792-7995$ \\
TNF & 6.7 & $1.97-16.94$ & 12.2 & $1.73-42$ \\
Mean ratio & 1.05 & $0.06-9.26$ & 0.29 & $0.06-1.74$ \\
Ang-2/Ang-1 & & & & $<0.003$ \\
\hline
\end{tabular}

angiogenic cascade, although the exact mechanism remains elusive. Previous research we have undertaken has associated elevated serum angiopoietin-2 (Ang-2) levels with angiodysplasia. Ang-1 and Ang-2 are ligands of the endothelial receptor tyrosine kinase Tie-2. Ang-1 regulates endothelial cell survival and blood vessel maturation and plays a key role in maintaining vascular integrity. Ang-2 is a functional antagonist of Ang-1. Inflammation and angiogenesis are associated with several pathological disorders and previous data suggests a TNF- $\alpha$ dependent dual functional roles of Tie2 in inflammatory angiogenesis

Methods Following informed consent, serum samples were collected from patients with a definite diagnosis of sporadic small bowel angiodysplasia (P2) on capsule endoscopy, and from healthy controls in which GI bleeding had been out-ruled by a negative faecal immunochemical test. Serum levels of Ang-1, Ang- 2 and TNF- $\alpha$ were measured using commercially available ELISA kits. All results were expressed as a mean and compared between patients and controls, and the mean of the ratio of ang2/ang1 levels for each group was calculated.

Results A total of 80 samples were analysed for each factor, including 40 patients ( $48 \%$ male, average age 71 years) and 40 controls (43\% male, average age 70 years). As expected and in keeping with our previous work levels of Ang-2 were significantly higher in patients (mean $4600 \mathrm{pg} / \mathrm{ml}$ ) than in controls (mean $2973 \mathrm{pg} / \mathrm{ml}$ ) p < 0.001. In addition levels of Ang-1 were significantly lower in the patient group (mean $13071 \mathrm{pg} / \mathrm{ml}$ ) vs. controls (mean $21169 \mathrm{pg} / \mathrm{ml}$ ) $\mathrm{p}<0.004$ (Table 1). We also found that levels of TNF- $\alpha$ were significantly lower in the patient group (mean $6.7 \mathrm{pg} / \mathrm{ml}$ ) vs. controls (mean $12.2 \mathrm{pg} / \mathrm{ml}$ ) p $<0.003$. The mean of the ratio of Ang2/Ang1 levels was found to be significantly higher in patients (1.05) vs. controls $(0.29) \mathrm{p}<0.05$.

Conclusion Ours is the first study to have identified a link between angiopoietin 1 and 2 ratios and gastrointestinal angiodysplasia. The TNF- $\alpha$ findings are also novel and would strongly suggest a role for inflammatory mediated angiogenesis in this condition.

Disclosure of Interest None Declared.

\section{PTH-139 LONG ACTING OCTREOTIDE THERAPY HAS A BENEFICIAL EFFECT IN PATIENTS WITH SIGNIFICANT SMALL BOWEL ANGIODYSPLASIA}

${ }^{1,2} \mathrm{G}$ Holleran*, ${ }^{3} \mathrm{~B}$ Hall, ${ }^{2} \mathrm{~N}$ Breslin, 'D McNamara. 'Department of Clinical Medicine, Trinity College Dublin, Tallaght, Ireland; ' Gastroenterology, Tallaght Hospital, Tallaght, Ireland; ${ }^{3}$ Trinity College Dublin, Tallaght, Ireland

\subsection{6/gutjnl-2014-307263.585}

Introduction Angiodysplasias account for up to 50\% of causes of small bowel bleeding and due to their relative inaccessibility and the intermittent nature of their bleeding they present a particular therapeutic challenge. Endoscopic ablation with APC is the most efficacious at reducing re-bleeding rates, however; its effect is short-lived and not all small bowel lesions are amenable to treatment via DBE. It has been suggested by a recent small trial that long-acting Octreotide may be beneficial at reducing re-bleeding rates.

\begin{tabular}{|c|c|c|c|}
\hline \multicolumn{4}{|c|}{ Abstract PTH-139 Table 1} \\
\hline & Hb pre-Rx & Most recent $\mathrm{Hb}$ & Re-bleeding episodes \\
\hline Overall & $8.7 \mathrm{~g} / \mathrm{dL}$ & $11.2 \mathrm{~g} / \mathrm{dL}$ & 5 \\
\hline Octreotide only & $8.6 \mathrm{~g} / \mathrm{dL}$ & $11.1 \mathrm{~g} / \mathrm{dL}$ & 2 \\
\hline APC and Octreotide & $9 \mathrm{~g} / \mathrm{dL}$ & $11.5 \mathrm{~g} / \mathrm{dL}$ & 3 \\
\hline
\end{tabular}

Methods Following ethical approval patients with significant recurrent anaemia ( $\geq 6$ months with RCC transfusions) and definite small bowel angiodysplasia (P2), on capsule endoscopy were invited to participate in an open label, uncontrolled pilot study of monthly $20 \mathrm{mg}$ long acting Octreotide. Patients who had lesions amenable to APC via DBE were treated prior to commencing Octreotide. Baseline demographics, medications, endoscopy and capsule findings and $\mathrm{Hb}$ level were recorded. Patients were assessed at regular intervals and evaluated for side effects and episodes of bleeding (defined as either overt bleeding or a $\mathrm{Hb}$ drop of $>1 \mathrm{~g} / \mathrm{dL}$ ). Patients who received fewer than three doses were excluded from analysis.

Results A total of 22 patients, 50\% $(\mathrm{n}=11)$ female, mean age of 72 years (range 55-92) have been recruited to date, $16(73 \%)$ with isolated small bowel angiodysplasias and 6 with small bowel and colonic lesions. Of these, 17 (77\%) received at least 3 doses, $11(65 \%)$ had DBE and APC prior to commencing Octreotide, and $11(65 \%)$ were on anticoagulants (4 warfarin, 7 aspirin and 2 dual antiplatelet therapy with aspirin and clopidogrel). The mean duration of treatment was 8.1 months (range 3-15). There was a statistically significant difference in mean baseline and follow up $\mathrm{Hb}$ levels, $8.7 \mathrm{~g} / \mathrm{dL}(5-11.1)$ and $11.2 \mathrm{~g} / \mathrm{dL}(8-14.3)$ respectively, $\mathrm{p}$ $<0.001$. Overall there were 8 re-bleeding episodes in 5 patients (29\%). Prior APC treatment did not affect outcome (Table 1). Significant side effects occurred in 3 patients (13.6\%), 1 allergic skin reaction, 1 thrombocytopenia and 1 choledocholithiasis.

Conclusion Our study has shown that long acting intramuscular Octreotide is effective at improving $\mathrm{Hb}$ levels in patients with refractory small bowel angiodysplasias. A longer follow up interval will be required to determine the beneficial effect of combination therapy. We have identified a relatively high rate of serious side effects, in contrast to previous reports, which need to be taken into consideration prior to commencing treatment.

Disclosure of Interest None Declared.

\section{PTH-140 SOLUBLE PLANTAIN FIBRE INHIBITS THE EPITHELIAL ADHESION OF DIARRHOEAL PATHOGENS CLOSTRIDIUM DIFFICILE, SALMONELLA AND ENTEROTOXIGENIC E. COLI (ETEC) THROUGH INTERACTION WITH THE INTESTINAL EPITHELIUM}

${ }^{1} \mathrm{H}$ Simpson*, ${ }^{2} \AA$. V. Keita, ${ }^{2} \mathrm{JD}$ Söderholm, ${ }^{1} \mathrm{JM}$ Rhodes, 'BJ Campbell. ${ }^{1}$ Gastroenterology, University of Liverpool, Liverpool, UK; ${ }^{2}$ Clinical and Experimental Medicine, Linkoping University, Linkoping, Sweden

10.1136/gutjnl-2014-307263.586 